\title{
Profit Sharing in Service Composition
}

\author{
Shigeo Matsubara \\ Department of Social Informatics, Kyoto University, \\ Yoshida-honmachi, Sakyo-ku, Kyoto 606-8501, Japan \\ matsubara@i.kyoto-u.ac.jp \\ http://www.ai.soc.i.kyoto-u.ac.jp/ matsubara
}

\begin{abstract}
Component services are often provided by different organizations, which needs to determine how to divide the profit obtained for the composite service to the component service providers. Previous studies have mainly focused on the process of aggregating multiple component services into a composite service. However, the process of the profit sharing has not yet discussed sufficiently. This problem can be formalized as a coalition game in the game theory. However, its flexibility of defining the policy of utilizing the services causes a problem. This paper shows that the existing profit sharing methods, more precisely, neither the equal division method nor the division method based on the Shapley value cannot satisfy the following two desiderata; (1) the sufficient level of service provision is attained, and (2) component services are not broken up more than is necessary. Moreover, we examine what factors make difficult to attain the sufficient level of service provision, and give a discussion toward mitigating this problem.
\end{abstract}

Keywords: Game theory, Shapley value, Incentive problem, Web services.

\section{Introduction}

An essential part of service-oriented computing technologies is service composition, i.e., a user's task is accomplished by aggregating multiple component services into a single composite service. Service composition has been actively studied 41] but the automated composition is still far from being achieved. We consider one of the reasons exists in lacking a sufficient discussion about an incentive problem, although recently incentive issues are getting more attention among the service-oriented computing researchers [25].

It seems that previous studies naively assumed that services are voluntarily provided and discussed how to find appropriate services among a huge amount of services. Here, voluntarily does not mean that services are provided with no charge but means that service providers do not strategically restrict the use of their services. However, as shown below, service providers may be motivated to impose a restriction on the use of their services.

A simple method of pricing a composite service is that the service providers set the prices of their own services individually, calculate the sum of each component 
service included in the composite service, and charge it to the user as a price of the composite service. The providers, however, are often difficult to set optimal prices that maximize their profit. This is because the providers are difficult to know in advance how their services are combined with other services.

A solution to overcome this drawback is to first set the price of the composite service that is equivalent to the user's valuation and then divide the profit to the component service providers included in the composite service. This paper focuses on how to solve a profit sharing problem.

Profit sharing problems are formalized as coalitional game in the game theory [3]. The coalitional game theory assumes that the members included in a coalition are disjoint with each other. That is, it assumes that any combinations of the component services are available if these component services are provided. However, such an assumption does not hold in the domain of web services. The service provider of $s_{1}$ can set the term of use in that utilizing $s_{1}$ in a composite service $s_{1} s_{2} s_{3}$ is allowed but utilizing $s_{1}$ in another composite service $s_{1} s_{3}$ is not allowed 1

Thus, we have to examine how this flexibility affects the profit sharing problem. More precisely, this paper examines whether the requirements for profit sharing in Web services are satisfied in the existing profit sharing methods such as equal division, profit sharing based on the Shapley value. We show that these methods cannot attain the sufficient level of service provision, examine what factors deteriorate the level of service provision, and finally give a discussion toward mitigating this problem.

\section{Model}

In this section we formalize the problem of profit sharing. $n$ service provider agents $S=\left\{s_{1}, s_{2}, \cdots, s_{n}\right\}$ and service user agents exist. To keep the discussion simple, we assume that a service provider agent provides a single component service. Thus, the set $S$ can be viewed as the set of component services as well as the set of service provider agents. A composite service can be composed from any subset $S_{i}$ of $S$ if it is allowed.

The users have their valuation $v: 2^{n} \rightarrow \Re$ for component services and composite services, which is called as characteristic function, where, $\Re$ is a set of real values and $v(\emptyset)=0$. This paper assumes that the valuation values are given for all the combinations of component services. In composing services by sequencing component service $s_{i}$ and component service $s_{j}$, it may happen that the type of output in $s_{i}$ does not compatible with the type of input in $s_{j}$. In such a case, we just let the valuation of the composite service to zero.

This paper assumes that zero monotonicity for the characteristic function. Zero monotonicity means that the value of a composite service gets larger as the number of component services included in the composite service increases. The assumption of zero monotonicity does not mean that users should choose the

${ }^{1}$ In this paper, $s_{1} s_{2} s_{3}$ represents a composite service, while $s_{1}, s_{2}, s_{3}$ represent a set of component services. 
services including more components of services. Users have a budget constraint and it may happen that users choose a low-price service, although its quality of service is not high.

The relevant characteristics of a user agent are summarized in that user's type $\theta_{i}=\left(v_{i}, b_{i}\right)$ where $v_{i}$ is the characteristic function and $b_{i}$ is the budget constraint. We assume that the distribution function of the user's type is known. From this assumption the profit for any combinations of the composite services can be calculated if the prices for each component service and each composite service are given. In addition, this paper does not consider the user's strategic behavior.

As mentioned above, a characteristic of web service is that service providers can flexibly set the term of use of their services. $s_{1} s_{2}$ represents a composite service that each component service is also available, while $\left[s_{1} s_{2}\right]$ represents a composite service that each component service is not available.

\section{Desiderata for Profit Sharing Methods}

Defining appropriate guiding principles is important in developing, maintaining, and utilizing the SOA. Granularity and composability have been discussed as design principles. Incentive issues, however, have not been examined sufficiently. Incentive issues become very significant especially in applying the SOA concept to inter-organizational services domain.

This paper considers the two requirements for profit sharing methods: (1) a variety of component services are provided to meet the user's requirements, (2) the unit of component services is appropriate, that is, a unit service is not too fine-grained. For example, we consider the service is too fine-grained if a dictionary service is divided to services of having indexes $\mathrm{A}, \mathrm{B}, \mathrm{C}$, and so on. If a unit of service is too fine-grained, the computational burden of calculating service composition appears.

In the rest of this section, we explain why under-provision of services should be considered. It happens that more than one Web services provided by different providers collude with each other and provide only a composite service, that is, do not provide each component service to users. Satisfying the sufficient provision of component services means that component services are provided in the manner that users are allowed to combine these services to other services to achieve the user's task.

Here, note that we do not intend to discuss the one-time usage of the services by service users. Consider user 1 and user 2 exist. If the times of utilizing the services are different, the identity of user 1 might be equal to the identity of user 2 . user 1 wants to use composite service $s_{1} s_{2} s_{3}$ and user 2 wants to use composite service $s_{1} s_{3}$. Here, if component service $s_{1}$ allows users to use $s_{1}$ as $s_{1} s_{2} s_{3}$ but does not allow users to use $s_{1}$ as $s_{1} s_{3}$, under-provision of services occurs. From the viewpoint of $u s e r 1$ it is sufficient if user 1 can use composite service $s_{1} s_{2} s_{3}$. This can be dealt with in the ordinal cooperative game theory, i.e., the profit sharing methods such as the Shapley values can be applied. However, if user 2 exists, whether using $s_{1} s_{3}$ is allowed or not should be considered simultaneously. 
Discussion on this paper is different from that about coalitional rationality in the cooperative game theory. For example, the collusion by $s_{1}$ and $s_{2}$ does not mean that $s_{1}$ and $s_{2}$ deviate the coalition of $s_{1} s_{2} s_{3}$ and create a new coalition including only $s_{1}$ and $s_{2} . s_{1}$ and $s_{2}$ will not reject to form a coalition of $s_{1} s_{2} s_{3}$ if it brings larger profit to $s_{1}$ and $s_{2}$. Here, collusion means that $s_{1}$ and $s_{2}$ reject to be included in the composite service $s_{1} s_{3}$ nor $s_{2} s_{3}$.

This discussion is caused from the fact that the granularity of the service is not clear. In the profit distribution problem in the game theory, the unit of player(agent) has no ambiguity. For example, in dealing with the problem of corporate alliance, a player represents a company.

We introduce the term of a level of service provision for the later discussions. A level of service provision means to what extent users are allowed to arbitrary combine these services with each other to accomplish their tasks.

\section{Drawbacks in the Existing Profit Sharing Methods}

This section proves that equal-division method and profit sharing method based on the Shapley value do not satisfy the requirements for the profit sharing in Web services.

\subsection{Profit Sharing by Equal Division}

A simple method for profit sharing is to share the profit for the composite service equally among the component service providers. This method is simple but has a drawback. Consider the following example. Two component services of $s_{1}, s_{2}$ and a composite service $s_{1} s_{2}$ exist. The characteristic functions are $v\left(s_{1}\right)=8$, $v\left(s_{2}\right)=2, v\left(s_{1} s_{2}\right)=12$. Here, if the profit is equally divided, $s_{1}$ and $s_{2}$ earn $12 / 2=6$, respectively. However, if the cost for providing component service of $s_{1}$ is $7, s_{1}$ suffers a loss. On the other hand, if $s_{1}$ provides its service as a single service, its profit becomes 8 , i.e., $s_{1}$ can make a money. Therefore, even if users want to use the composite service of $s_{1} s_{2}, s_{1}$ will prevent it.

\subsection{Profit Sharing by Using the Shapley Value}

Next, we consider to use the Shapley value. The Shapley value has been studied as a profit/cost sharing method [3]. The Shapley value represents the marginal contribution of component service $s_{i}$, i.e., to what extent the user's valuation increases by introducing $s_{i}$ to the existing service $S_{i}-s_{i}$.

$$
\phi_{s_{i}}=\sum_{S_{i}: s_{i} \in S_{i} \subset S} \frac{\left(\# S_{i}-1\right) !\left(n-\# S_{i}\right) !}{n !}\left\{v\left(S_{i}\right)-v\left(S_{i}-s_{i}\right)\right\}
$$

Here, $\# S_{i}$ represents the number of component services included in $S_{i}$ and $v(\emptyset)=0$.

The Shapley value satisfies the desirable properties such as Pareto optimality, the null player property, the equal treatment property, and additivity. However, 
it causes a problem if we apply it to the profit sharing problem among Web services because the unit of services is not obvious.

Consider the following example. Three component services of $s_{1}, s_{2}, s_{3}$ exist and the characteristic function is given as $v\left(s_{1}\right)=v\left(s_{2}\right)=v\left(s_{3}\right)=0, v\left(s_{1} s_{2}\right)=5$, $v\left(s_{2} s_{3}\right)=3, v\left(s_{1} s_{3}\right)=3, v\left(s_{1} s_{2} s_{3}\right)=10$. The Shapley values are calculated as follows. $\phi_{s_{1}}=11 / 3, \phi_{s_{2}}=11 / 3$, and $\phi_{s_{3}}=8 / 3$.

Next, consider the case that services providers of $s_{1}$ and $s_{2}$ do not provide $s_{1}$ and $s_{2}$ as a component service but only provide a composite service of $\left[s_{1} s_{2}\right]$. Here, users are not allowed to use the composite services of $\left[s_{1} s_{3}\right]$ nor $\left[s_{2} s_{3}\right]$. The characteristic function is given as $v\left(\left[s_{1} s_{2}\right]\right)=5, v(c)=0, v\left(\left[s_{1} s_{2}\right] s_{3}\right)=10$. In this case, the Shapley values are calculated as $\phi_{\left[s_{1} s_{2}\right]}=15 / 2$ and $\phi_{s_{3}}=5 / 2$.

Compared the two cases, $s_{1}$ and $s_{2}$ can earn $11 / 3+11 / 3=22 / 3$ if $s_{1}$ and $s_{2}$ are provided as component services, while they can earn 15/2 if $s_{1}$ and $s_{2}$ do not provide $s_{1}$ nor $s_{2}$ as a component service. The profit in the latter case is larger than that in the former case, which means that users cannot use the composite service of $\left[s_{1} s_{3}\right]$ nor $\left[s_{2} s_{3}\right]$. This means that a virtue of service computing that a variety of services are provided and users can arbitrarily combine these services to satisfy their demands is spoiled. The next section examines the conditions that providers choose a strategy of not providing a component service even if they can do it.

\section{Analysis of the Levels of Service Provision}

This section discusses the profit sharing based on the Shapley values. We draw the conditions in the case including only three component services, and then, we analyzes what factors affect the levels of service provision.

\subsection{Case of Thee Component Services}

This section discusses the case of three component services available. If $s_{1}, s_{2}, s_{3}$ are provided by each provider, the Shapley values are calculated as follows.

$$
\begin{aligned}
s_{1}: & \frac{1}{6}\left(v\left(s_{1}\right)+v\left(s_{1}\right)+v\left(s_{1} s_{2}\right)-v\left(s_{2}\right)+v\left(s_{1} s_{3}\right)-v\left(s_{3}\right)+v\left(s_{1} s_{2} s_{3}\right)-v\left(s_{2} s_{3}\right)+\right. \\
& \left.v\left(s_{1} s_{2} s_{3}\right)-v\left(s_{2} s_{3}\right)\right) \\
s_{2}: & \frac{1}{6}\left(v\left(s_{2}\right)+v\left(s_{2}\right)+v\left(s_{1} s_{2}\right)-v\left(s_{1}\right)+v\left(s_{2} s_{3}\right)-v\left(s_{3}\right)+v\left(s_{1} s_{2} s_{3}\right)-v\left(s_{1} s_{3}\right)+\right. \\
& \left.v\left(s_{1} s_{2} s_{3}\right)-v\left(s_{1} s_{3}\right)\right)
\end{aligned}
$$

Therefore, $s_{1}$ and $s_{2}$ obtain the following profit in total.

$$
\frac{1}{6}\left(v\left(s_{1}\right)+v\left(s_{2}\right)-2 v\left(s_{3}\right)+2 v\left(s_{1} s_{2}\right)-v\left(s_{2} s_{3}\right)-v\left(s_{1} s_{3}\right)+4 v\left(s_{1} s_{2} s_{3}\right)\right)
$$

Next, if $s_{1}$ and $s_{2}$ collude with each other and $\left[s_{1} s_{2}\right]$ is provided as a component service, the profit share is calculated as follows.

$$
\frac{1}{2}\left(v\left(s_{1} s_{2}\right)+v\left(s_{1} s_{2} s_{3}\right)-v\left(s_{3}\right)\right)
$$


By calculating the difference between the expressions of (11) and (2), we obtain the followings.

$$
\frac{1}{6}\left(v\left(s_{1}\right)+v\left(s_{2}\right)+v\left(s_{3}\right)-v\left(s_{1} s_{2}\right)-v\left(s_{2} s_{3}\right)-v\left(s_{1} s_{3}\right)+v\left(s_{1} s_{2} s_{3}\right)\right)
$$

Therefore, if the following inequality holds, the composite services of $s_{1} s_{3}$ and $s_{2} s_{3}$ are available for users.

$$
v\left(s_{1} s_{2} s_{3}\right)+v\left(s_{1}\right)+v\left(s_{2}\right)+v\left(s_{3}\right)>v\left(s_{1} s_{2}\right)+v\left(s_{2} s_{3}\right)+v\left(s_{1} s_{3}\right)
$$

Whether component services are provided so that any combinations of these component services are allowed can be affected by the following two strategic behaviors. One is to eliminate the contribution of other composite service $\left(s_{1} s_{3}\right.$, $s_{2} s_{3}$ in the above example) and increase the own contribution of $\left(s_{1} s_{2}\right)$ relatively by providing it as a single service. Another is to increase the profit share by providing the component service without any restrictions and increasing the number of share holders.

When the user's valuation considerably increases if the three services of $s_{1}$, $s_{2}, s_{3}$ are used as a set, the share of $s_{1}$ and $s_{2}$ increase by individually having the share. On the other hand, if the user's valuation for $s_{1} s_{2} s_{3}$ is not so larger than $s_{1} s_{2}, s_{1} s_{3}, s_{2} s_{3}, s_{1}$ and $s_{2}$ can increase their share by preventing each of $s_{1}$ and $s_{2}$ as a component service.

If the number of the component services becomes more than three, the problem becomes more complicated. This is because the collusion by the three providers as well as the collusion by the two providers may occur. Here, we have a question on what conditions the levels of service provision is spoiled. The next section examines how the number of providers affects the levels of service provision.

\subsection{Effects of the Number of Providers}

Suppose that a composite service of $X$ including $k$ component services and $X$ is provided as a composite service, i.e., a subset of component services in $X$ are not provided to users. In addition, to make analysis tractable, we assume that the composite services are homogeneous, which means that the characteristic function is symmetric to any component services. Here, we can say that the value of the characteristic function can be determined by the number of component services $i$ included in the composite service. We designate the value per component service as $v_{i}\left(v_{1}<v_{2}<\cdots<v_{n}\right)$ in the case that $i$ component services are included.

Here, if component services of $X$ and $y$ exist, the characteristic function can be represented as follows.

$$
v(X y)=(k+1) v_{k+1}, v(X)=k v_{k}, v(y)=v_{1}
$$

The share of $X$ is given as follows.

$$
\frac{1}{2}\left(k v_{k}+(k+1) v_{k+1}-v_{1}\right)
$$


On the other hand, if the component services included in $X$ are provided individually, the profit share of each service can be given by dividing the profit $(k+1) v_{k+1}$ for providing the composite service $X y$ equally to $(k+1)$ providers because we assumes that the component services are homogeneous. Thus, the increase of the profit share of each component service by providing $X$ as a component service can be calculated as follows.

$$
\frac{1}{2}\left(v_{k}-\frac{k-1}{k} v_{k+1}-\frac{1}{k} v_{1}\right)
$$

This expression tells that the increase becomes less than zero if $k$ increases. Service providers can claim that the set of their services is more valuable by colluding each other and preventing users from using any combinations of these services. However, the number of $k$ further increases, the profit has to be shared by the more providers, which results in reducing the profit share increase by the collusion.

\section{Toward Problem Solving}

To overcome the drawbacks that applying the profit sharing method based on the Shapley values to the service computing domain spoils the sufficient service provision, we propose the direction of dealing with the problem. The idea is to give the same share as that obtained by forming a collusion if the collusion gives a larger amount of profit, which discourage providers to collude with each other.

In the example in section 4.2, we showed that $s_{1}$ and $s_{2}$ have an incentive to collude with each other and provide $s_{1} s_{2}$ as a component service. Here, if $s_{1}$ and $s_{2}$ are paid $15 / 2$, they do not have an incentive not to provide $s_{1}$ or $s_{2}$ as a component service, which enables users to utilize composite services of $s_{1} s_{3}$ or $s_{2} s_{3}$. The profit share of $15 / 2$ between $s_{1}$ and $s_{2}$ are determined by calculating the Shapley values, although $s_{1}$ and $s_{2}$ share equally $15 / 4$ each because we assume services are homogeneous. The share of component service $s_{3}$ decreases to $5 / 2$. However, its share is larger than zero that is the share in providing $s_{3}$ without combining other services.

As mentioned above, if the number of component services is more than three, a various type of collusions may exist. Here, we have a good news that we do not have to examine a case of including quite a many providers because the number of component services becomes large, the providers are less motivated to collude with each other. Carrying out more detained analysis is included in our future work.

So far, we have discussed how to maintain the sufficient level of service provision. However, satisfying only the sufficient level of service provision is not sufficient in the context of incentive. This is because providers may have an incentive to break a service into many of too fine-grained component service as explained in Section [3. For example, a provider of operating dictionary service can break the dictionary service into sub services of having only index-a entries, index-b entries, etc. if it brings the provider larger profit. 
A method to solve this problem is to deal with a set of services as a component service if these services are provided by a single provider. This prevents too finegrained services to be provided.

\section{Concluding Remarks}

This paper pointed out that neither the adding-up method nor the equal-division method have drawback in sharing the profit among the providers in the serviceoriented computing domain. Next, we introduced the concept of a level of service provision, which means to what extent users are allowed to arbitrary combine these services with each other to accomplish their tasks. We showed that the profit sharing method based on the Shapley values, which has been studied in the game theory, cannot attain the sufficient level of service provision. The analysis showed that the level of service provision gets worse if the number of the component services included in the corresponding composite service is small. In addition, we gave a direction to solve this problem.

The discussion shows a possibility of solving the problem of service-oriented computing by introducing the game theory. On the other hands, service-oriented computing might be possible to affect the studies of the Shapley values. So far, the research efforts have been invested in clarifying the axioms of the Shapley values in the game theory and in dealing with the computational complexity of calculating the Shapley values for a large-scale problem in computer science. Here, the flexibility of defining the policy of service utilization causes a new type of collusion. Further investigation about this problem is included in our future work.

Acknowledgments. This research was partially supported by a Grant-in-Aid for Scientific Research (B) (22300052, 2010-2012) and a Grant-in-Aid for Scientific Research (A) (21240014, 2009-2011) from Japan Society for the Promotion of Science (JSPS).

\section{References}

1. Ben Hassine, A., Matsubara, S., Ishida, T.: A Constraint-Based Approach to Horizontal Web Service Composition. In: Cruz, I., Decker, S., Allemang, D., Preist, C., Schwabe, D., Mika, P., Uschold, M., Aroyo, L.M. (eds.) ISWC 2006. LNCS, vol. 4273, pp. 130-143. Springer, Heidelberg (2006)

2. Cheng, H.K., Tang, Q.C., Zhao, J.L.: Web services and service-oriented application provisioning: An analytical study of application service strategies. IEEE Transactions on Engineering Management 53(4), 520-533 (2006)

3. Shapley, L.S.: A value for n-person games. Annals of Mathematical Studies 28, 307-317 (1953)

4. Sirin, E., Parsia, B., Wu, D., Hendler, J., Nau, D.: HTN planning for web service composition using shop2. Journal of Web Semantics 1(4), 377-396 (2004)

5. Zheng, X., Martin, P., Powley, W., Brohman, K.: Applying bargaining game theory to web services negotiation. In: Proceedings of the 2010 IEEE International Conference on Services Computing (SCC 2010), pp. 218-225 (2010) 